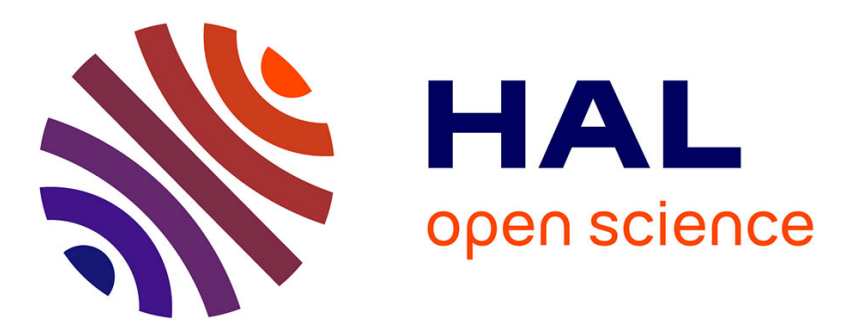

\title{
Assessing Early Cardiac Outflow Tract Adaptive Responses Through Combined Experimental-Computational Manipulations
}

\author{
Stephanie Lindsey, Irene Vignon-Clementel, Jonathan Butcher
}

\section{To cite this version:}

Stephanie Lindsey, Irene Vignon-Clementel, Jonathan Butcher. Assessing Early Cardiac Outflow Tract Adaptive Responses Through Combined Experimental-Computational Manipulations. Annals of Biomedical Engineering, 2021, 49 (12), pp.3227-3242. 10.1007/s10439-021-02802-2 . hal-03523736

\author{
HAL Id: hal-03523736 \\ https://hal.science/hal-03523736
}

Submitted on 12 Jan 2022

HAL is a multi-disciplinary open access archive for the deposit and dissemination of scientific research documents, whether they are published or not. The documents may come from teaching and research institutions in France or abroad, or from public or private research centers.
L'archive ouverte pluridisciplinaire HAL, est destinée au dépôt et à la diffusion de documents scientifiques de niveau recherche, publiés ou non, émanant des établissements d'enseignement et de recherche français ou étrangers, des laboratoires publics ou privés. 
Published in Annals of Biomedical Engineering, 2021 - https://doi.org/10.1007/s10439-021-02802-2

Supplementary movies and files available on the journal website: https://link.springer.com/article/10.1007/ s10439-021-02802-2

\section{Assessing Early Cardiac Outflow Tract Adaptive Responses Through Combined Experimental-Computational Manipulations}

Stephanie E. Lindsey, Ph.D. ${ }^{1 \#, ~ I r e n e ~ E . ~ V i g n o n-C l e m e n t e l, ~ P h . D . ~}{ }^{\star}$, and Jonathan T. Butcher, Ph.D. ${ }^{1 *}$

${ }^{1}$ Nancy E. and Peter C. Meinig School of Biomedical Engineering ${ }^{2}$ Centre de Recherche Saclay-IDF, Palaiseau, France

*This collaborative study is accomplished by joint corresponding senior authors (IVC and JTB).

\#Current address: Mechanical and Aerospace Engineering, UC San Diego, La Jolla, CA 92093

Address for Correspondence:

Irene Vignon-Clementel, PhD

Directeur de Recherche Inria

Centre de Recherche Inria de Saclay-IDF

rue Honoré d'Estienne d'Orves

91120 Palaiseau (FRANCE)

Phone: +33(0) 1.80.49.42.67

E-mail: irene.vignon-clementel@inria.fr

AND

Jonathan T. Butcher, PhD

Professor

Nancy E. and Peter C. Meinig School of Biomedical Engineering

Cornell University

304 Weill Hall

Ithaca, NY 14853-7202 (USA)

Tel: 607.255 .3575

Fax: 607.255 .7330

E-mail: jtb47@cornell.edu 


\section{Abstract:}

Mechanical forces are essential for proper growth and remodeling of the primitive pharyngeal arch arteries (PAAs) into the great vessels of the heart. Despite general acknowledgement of a hemodynamic-malformation link, the direct correlation between hemodynamics and PAA morphogenesis remains poorly understood. The elusiveness is largely due to difficulty in performing isolated hemodynamic perturbations and quantifying changes in-vivo. Previous in-vivo arch artery occlusion/ablation experiments either did not isolate the effects of hemodynamics, did not analyze the results in a 3D context or did not consider the effects of varying degrees of occlusion. Here, we overcome these limitations by combining minimally invasive occlusion experiments in the avian embryo with 3D anatomical models of development and in-silico testing of experimental phenomenon. We detail morphological and hemodynamic changes twenty-four hours post vessel occlusion. 3D anatomical models showed that occlusion geometries had more circular cross-sectional areas and more elongated arches than their control counterparts. Computational fluid dynamics revealed a marked change in wall shear stress-morphology trends. Instantaneous (in-silico) occlusion models provided mechanistic insights into the dynamic vessel adaptation process, predicting pressure-area trends for a number of experimental occlusion arches. We follow the propagation of small defects in a single embryo Hamburger Hamilton $(\mathrm{HH})$ Stage 18 embryo to a more serious defect in an $\mathrm{HH} 29$ embryo. Results demonstrate that hemodynamic perturbation of the presumptive aortic arch, through varying degrees of vessel occlusion, overrides natural growth mechanisms and prevents it from becoming the dominant arch of the aorta. 


\section{Introduction:}

During cardiac morphogenesis, blood exits the developing chick embryo's heart through the pharyngeal arch artery system. These six bilateral paired vessels exist in various combinations with the fifth arch existing as a fleeting bypass of the sixth arch. The arches sequentially emerge, remodel and disappear before forming the mature aortic arch, pulmonary artery, pulmonary veins and venae cavae. Hemodynamics plays a vital role in the maturation of the pharyngeal arch artery system ${ }^{15,18,21,35}$. The role of abnormal hemodynamics in the creation of cardiac abnormalities has intrigued researchers for decades $4,8,12-15,27,29,30$. Disruption of established flow patterns during critical windows of development produces a range of defects that drastically alter function of the mature heart. These defects may stem from improper cardiac looping, incomplete outflow tract rotation, incomplete outflow tract septation, abnormal maturation of the cardiac valves or yet to be determined factors. Malformations of the outflow tract account for over $50 \%$ of clinically relevant congenital heart defects ${ }^{7}$. Determining the origin of such defects continues to pose a major challenge to the field. Cardiac morphogenesis is a complex interconnected process that is difficult to delineate. Altered hemodynamic flow patterns in the heart following surgical manipulation of the atrium, have been shown to affect the ventricle, as well as the pharyngeal arch artery system. Ligation of the left atrium redistributes flow to the right side of Hamburger Hamilton $(\mathrm{HH})$ Stage 21 embryos, immediately affecting flow patterns and hemodynamic forces in the arch arteries. Within 24 hours of left atrial ligation, the fourth aortic arch of $\mathrm{HH} 24$ embryos is drastically reduced in dimensions. By 
HH27 (48 hours post ligation) arches were skewed and distorted. All embryos surviving to $\mathrm{HH} 34$ (day 8) displayed hypoplastic ventricles; $70 \%$ also possessed abnormal aortic $\operatorname{arch}$ patterning ${ }^{15}$.

While much has been gained from ligation experiments such as those performed by Hu et al., a major limitation in determining the causality of clinically relevant cardiac abnormalities is the difficulty of studying the effects of hemodynamics alone in the creation of cardiac abnormalities. In an effort to surgically manipulate flow patterns in experimental animal models, researchers have used a combination of ligations, clipping, and cauterizations, all of which disrupt flow patterns, as well as change the properties of the surrounding tissues. Alteration of mechanical stress and strains in the developing heart have been shown to regulate vascular growth and remodeling as well as trigger cell signaling and subsequent organization patterns ${ }^{5,9,14}$. Recent advances in imaging technology have allowed for the minimally invasive occlusion of flow in the developing vascular system ${ }^{21}$. Studies such as these may prove to be important in delineating the sequence of events that leads to clinically relevant cardiac abnormalities. The coupling of computational modeling with targeted experimental studies can provide further insight into the mechanisms behind abnormal cardiac morphogenesis, correlating sites of altered wall shear stress (WSS) with commonly affected areas in the clinically associated disease model ${ }^{18}$ or highlight driving forces in development ${ }^{21,36}$.

Another major limitation in determining causality for cardiac malformations is an inability to create, study or identify what likely begins as a subtle abnormality before propagating into clinically serious malformations. For this reason, we characterized normal PAA development in a cohort-based study that identified a range of normal 
development in Hamburger Hamilton $(\mathrm{HH})$ staged 18, 24, and 26 embryos, taking into account morphological and hemodynamic parameters and determining a relationship between the two ${ }^{20}$. In light of this work, we can quantify the deviation from normal exhibited in $\mathrm{HH} 24$ embryos upon $\mathrm{HH} 18$ vessel occlusion. Though our group has previously studied the effects of arch artery occlusion on $\mathrm{HH} 18$ and $\mathrm{HH} 24$ embryos ${ }^{21}$, the experimental results were not considered in 3D, which allows for a more thorough investigation of pathologies ${ }^{3}$. Additionally, our previous study did not consider the effect of partial occlusions. The objective of this study is therefore to understand the severity of varying degrees of vessel occlusions ${ }^{21}$ and assess the structural and functional changes taking place upon experimental occlusion. We focus on partial occlusions which are likely more representative of clinically serious congenital heart defects where the fetus is carried to term but the heart is malformed. The word 'occlusion' is used to refer to both full and partial vessel occlusions, with the distinction being made when necessary. Using detailed 3D reconstructions of experimental occlusion geometries, we analyze vessel adaption to abnormal hemodynamic loading that originates with the arch arteries themselves. We compare experimental results post-occlusion to that of in-silico occlusions taken at the time of intervention $(\mathrm{HH} 18)$ and 24-hours post intervention $(\mathrm{HH} 24)$ when the embryo's vasculature has had time to remodel and adapt (Fig. 1). We subsequently demonstrate how an embryo's "small deviation from normal" can propagate into a more serious malformation. Ultimately, this work details structural compensation mechanisms involved in early cardiac outflow morphogenesis and the effects on hemodynamic function and subsequent cardiac growth. 


\section{Methods:}

\section{Creation of In-vivo Occlusion Models}

Embryos were experimentally occluded as previously described ${ }^{21}$. Briefly, $\mathrm{HH} 18$ embryos were open cultured. Embryos with smaller IVR arch arteries were specifically chosen to facilitate faster occlusion experiments. Selected embryos were injected with Texas red dextran (70,000 MW, neutral Sigma-Aldrich D1830) diluted in Earle's balanced salt solution $(5 \% \mathrm{w} / \mathrm{v})$ so that their vasculature could be visualized by way of two-photon microscopy. A custom built two-photon excited fluorescence microscope with a separate femtosecond pulsed photoablation laser was used to perform targeted vessel disruption. The photoablation laser consisted of 1-kHz high-pulse-energy Ti:Sapphire amplified laser system with 50-fs pulse duration (Legend-USP, Coherent, Santa Clara, CA, 800-nm central wavelength). Photodisruption was controlled and confined to the volume focused by two-photon microscopy optics (see supplementary methods).

\section{Ultrasound processing and generation of computational inlet flow curves}

Outflow tract (OFT) velocity and that of the three paired pharyngeal arch arteries were measured using B-mode guided Doppler Ultrasound (Vevo770 and Vevo 2100, Visualsonics, Inc.). A Poiseuille OFT velocity profile was assumed to convert the OFT Doppler data into flow rate, and apply this velocity information as an inlet boundary condition for an initial 3D CFD simulation. This initial CFD simulation was used to obtain the velocity spatial profile in each of the arches and a new inlet flow (Qinlet) calculated to be the sum of flow in each of the individual arches, as previously described $^{20}$. 


\section{In-silico geometry preparation and 3D-0D flow modeling}

Embryo-specific 3D geometries of $\mathrm{HH} 18 / \mathrm{HH} 24$ controls and $\mathrm{HH} 24$ experimental occlusions were generated by importing nano-CT images into MIMICS (Materialise, Louvain, Belgium) and 3MATICs (Materialise, Louvain, Belgium). Geomagics Studio 10 (Geomagic Inc., Durham, NC) was also used for the preparation of 3D geometries for CFD. All embryos were scaled to account for the difference between dehydrated (see supplementary methods) and native vessel size as determined by a series of in vivo verifications that included cranial-rump and diameter measurements. India ink and Texas Red Dextran were used to obtain native vessel size across stages, results compared to that of $3 \mathrm{D}$ reconstructions, and a scaling factor was generated. Due to the rigid nonaggregated nature of red blood cells at these stages of development, blood was treated as an incompressible Newtonian fluid with constant hemodynamic properties ${ }^{37}(\rho=1060$ $\mathrm{kg} / \mathrm{m}^{3}, \mu=3.71 \times 10^{-3}$ Pa.s) and rigid, impermeable vessel walls were assumed with no slip boundary conditions. Pulsatile flow was simulated by solving the Navier-Stokes equations, on a high-resolution unstructured grid with finite-element numerical treatment (solved using the FELiSce library) (gforge.inria.fr/projects/felisce) ${ }^{25}$. Grid sensitivity analysis was conducted on a control PAA model for each day in order to ensure consistency and reliability of the numerical solutions for all simulations presented in this study, beyond which resulting mass-flow redistributions were insensitive to further Cartesian grid refinements.

\section{Boundary Conditions:}

A natural spatial velocity profile was imposed at the inlet. To obtain this an auxiliary steady Stokes equation, with natural boundary conditions at the outlets, was solved first. 
The resulting inlet velocity profile was subsequently scaled at each time-point to match the measured flow-rate Qinlet and imposed on full pulsatile flow simulations. RCR Windkessel models were imposed at the outlets to represent the downstream vasculature as measured by ${ }^{39}$. Windkessel parameters were tuned to assure the distribution of the cardiac output to dorsal aorta (DoA) and cranial vessels maintains a 90-10 flow split over the course of one cycle ${ }^{16}$ as summarized in the $0 \mathrm{D}$ section (see supplemental methods). The differential equation representing the RCR circuit is

$$
P+R_{5} C \frac{d P}{d t}=\left(R_{4}+R_{5}\right) Q+R_{5} R_{4} C \frac{d Q}{d t}
$$

Where $R_{4}$ is the proximal resistance, $R_{5}$ is the distal resistance, $C$ represents the capacitance, $P$ is pressure and $Q$ is flow (SFig. 1).

\section{Creation of In-Silico Occlusion Models}

In-silico occlusion models were made from two $\mathrm{HH} 18$ and two $\mathrm{HH} 24$ pharyngeal arch artery systems. The two $\mathrm{HH} 18$ control references $(\mathrm{HH} 18-1$ and $\mathrm{HH} 18-5)$ were chosen to represent the wide range of variability seen across $\mathrm{HH} 18$ embryos ${ }^{20}$. $\mathrm{HH} 18-1$ has a rapidly regressing right and left lateral second arches as well as a developing right and left lateral forth arch, while $\mathrm{HH} 18-5$ has the largest right and left lateral second arch, as well as the largest right and left lateral fourth arch, seen in our $\mathrm{HH} 18$ control subset (SFig. 1B). Similarly for HH24, two embryos (HH24-1 and HH24-4) were chosen to represent the natural range of pressure variation seen in controls and because $\mathrm{HH} 24-$ 1 exhibited right flow dominance, while $\mathrm{HH} 24-4$ exhibited left flow dominance ${ }^{20}$. A full occlusion and a partial occlusion were made for each of the four reference geometries, bringing the number of in-silico occlusion geometries to eight. Partial occlusions were made to mimic those seen in experimentally occluded embryos, either through two- 
photon microscopy (HH18 embryos) or their subsequent nano-CT reconstructions from images taken 24 hours post vessel occlusion.

\section{Statistical Analysis}

Morphological and hemodynamic changes were compared qualitatively and quantified when possible. Results were summarized in the form of mean and standard deviation values over the course of one cardiac cycle. Two-tailed two-sample t-tests were used when comparing between control and partial occlusion embryos with $p<0.05$ denoting significance. A one sample t-test was also used when comparing a single occlusion sample to that of controls. Linear regressions were performed on hemodynamic versus geometrical parameter graphs. Statistical comparisons were made through the use of GraphPad Prism (GraphPad Software, Inc San Diego, CA) statistical software.

\section{Results:}

\section{Early great vessel morphogenesis depends on hemodynamic flow patterns.}

To explore adaptive responses of the developing vasculature, mechanical loading was altered via in-vivo vessel occlusion. We occluded the developing IVR PAA in $\mathrm{HH} 18$ embryos using nonlinear optical techniques that allowed for changes in hemodynamics alone (see methods). To date, this is the only experimental method that locally and noninvasively alters flow patterns. Occlusion experiments were purposely performed on $\mathrm{HH} 18$ embryos and selected to have a narrow (nascent) PAA IV vessel in order to facilitate vessel occlusion. Although PAA IVR was small ( $\sim 30 \mu \mathrm{m}$ in diameter) at time of occlusion ( $\mathrm{HH} 18)$, by $\mathrm{HH} 24$ it carries approximately $22 \%$ of flow exiting the heart ${ }^{20}$. Following $\mathrm{HH} 18$ vessel occlusion, the largest percentage of flow was re-directed 
to PAA IIIL in the partial occlusion geometries and PAA VIL in the case of full vessel occlusion, with PAA IIIL carrying $35 \%$ of flow compared to $20 \%$ of flow in its control counterpart in partial occlusion geometries and PAA VIL carrying $31 \%$ of flow compared to $7 \%$ in its control counterpart in full occlusion geometries (Fig. 2A, STable1). Within 24 hours post-occlusion, embryos exhibited a range of structural defects (SFig. 2,3). Observed malformations include the merging of arch arteries into a single vessel before separating into two distinct vessels, abnormal arch artery spacing, skewed right and left branches, enlarged arch arteries and outflow inlets, abnormal rotation of the outflow tract (OFT) junction, abnormal bulging of the aortic sac and abnormal patterning of the cranial DoA branches. Position and length of the initial vessel occlusion were associated with certain types of defects. Occlusions occurring near the DoA junction were associated with merged vessel pathways originating from the aortic sac, while occlusions occurring near the aortic sac junction were associated with enlarged OFT orifices. More elongated vessel occlusions, that spanned more of the lateral length, were associated with abnormal spacing and skewed left and right patterning.

Regardless of initial occlusion placement, structural analysis of $\mathrm{HH} 24$ occlusion geometries revealed a lengthening along the centerline of partial occlusion embryos, with a significant change observed in PAA III, IV and VI right, when compared to those of controls (Fig. 3C). Arch arteries also became more circular in cross-section than their $\mathrm{HH} 24$ control counterparts, approaching the phenotype of an early HH26 embryo in both length and ellipticity, but maintaining a non-septated OFT (Fig. 3A, B). HH24 arch artery area largely increases in PAA IIIL in order to compensate for HH18 IVR vessel occlusion (Fig. 3D). Branch-splitting angle decreased for HH24 PAA IIIR and IIIL partial 
occlusion geometries when compared to that of $\mathrm{HH} 24$ controls (Fig. 3E). When comparing $\mathrm{HH} 24$ partial and full occlusion branch splitting angles, the largest changes exist for the right DoA aorta branch-splitting angle and PAA IIIL.

In-silico occlusion models of PAA development. To understand the observations described above, we developed an experimental-computational pipeline that begins by taking experimental-based computational models and re-distributing natural flow patterns through in-silico occlusion (Fig. 1). In isolating the immediate flow and pressure redistributions, one can see how the embryo's response differs from empirical predictions and begin to dissect the compensation mechanisms at work. Both full and partial occlusions were performed on $\mathrm{HH} 18$ and $\mathrm{HH} 24$ control geometries, which span the initial 24-hour remodeling period. Because between $\mathrm{HH} 18$ and $\mathrm{HH} 24$ one arch pair regresses (PAA II) and another becomes patent (PAA VI), the two in-silico models convey immediate changes for their respective arch configurations (Fig. 1). Boundary conditions were kept constant between control and in-silico occlusions, so as to highlight instantaneous flow redistribution when the vessel is not given time to adapt to changing hemodynamic forces. With only $5-7 \%$ of flow being re-distributed upon occlusion, changes in $\mathrm{HH} 18$ in-silico flow distribution were small (STable 2A,B). Upon PAA IVR vessel occlusion, more flow is re-distributed to the left side of the embryo than the right in the full occlusion case (approximately $59 \%$ of flow re-direction is channeled to the left), and is distributed equally in the case of the partial occlusion. The corresponding changes in pressure and wall shear stress (WSS) as measured by numerical simulation are subtle (Fig. 4B, top) with a slight increase in pressure magnitude visible at the aortic sac. Pressure and WSS magnitude in the left IV are 
slightly increased upon partial occlusion in both $\mathrm{HH} 18$ in-silico occlusion subsets. The results of both pressure and flow changes are summarized in Fig. 4A in the form of resistance $\left(\frac{\Delta P}{Q}\right.$, supplemental Eq 1.2$)$. HH18 PAA IVR resistance rapidly increases with the decreased flow rate caused by vessel partial occlusion and is infinite in the case of full vessel occlusion $(Q=0)$ when compared to that of controls, while resistance values for the other vessels remain largely unchanged.

Full and partial occlusions followed the same instantaneous flow re-distribution patterns, with PAA IIIL receiving $29 \%$ and $27 \%$ of the flow and PAA IVL receiving $24 \%$ and $23 \%$ of flow respectively in $\mathrm{HH} 24$ full and partial occlusion scenarios (Fig. 2A). Changes in HH24 in-silico occlusion pressure and WSS maps (Fig. 4B, bottom) are more pronounced than that of $\mathrm{HH} 18$ in-silico occlusions, but follow a similar pattern. Upon occlusion, pressure magnitude increased at the outflow tract inlet before dissipating cranially along the aortic sac, laterally along the IVR occluded or partially occluded arch as well as along the caudal VI arch arteries. Spikes in WSS increase in high pressure areas. These changes are summarized in the form of a dramatic increase in the resistance value of PAA IVR, small increase in PAA III (R \&L) and PAA IVL. PAA VIR remains unchanged and PAA VIL slightly decreases. In both $\mathrm{HH} 18$ and $\mathrm{HH} 24$ insilico occlusion scenarios, the embryo's immediate response was to send additional flow to PAA IVL. 3D numerical simulations of experimental occlusion embryos revealed that in reality in-vivo flow distribution to PAA IVL decreased in both the experimental partial occlusion and full occlusion geometries, suggesting that other mechanisms may also be at play. 
Overriding of PAA natural growth mechanisms. CFD of $\mathrm{HH} 24$ experimental occlusion embryos (24 hours post occlusion) allowed us to investigate the evolution of hemodynamic flow patterns and associated forces (STable3, experimental vs computational reference). Experimental partial occlusion pressure and WSS maps resemble that of control embryos while the full occlusion falls within the same range but has a more elevated pressure and WSS magnitudes along the DoA (Fig. 2B). The experimental occlusion embryo maintains the same general pressure dissipation pattern, but is unable to fully dissipate pressure before the arches converge at the DoA. To delineate the role of pressure and WSS as drivers of PAA remodeling, we quantified morphological changes from CFD-aided 3D renderings obtained from point-to point segment makers, as outlined in ${ }^{20}$. Statistically significant changes between relative position at $\mathrm{HH} 18$ to $\mathrm{HH} 24$ were found through linear regression of data obtained per arch. Linear regressions were performed using $\mathrm{HH} 18$ control data ( $\mathrm{N}=5$ embryos) or $\mathrm{HH} 18$ in-silico occlusion data ( $\mathrm{N}=2$ embryos) as time point 1 and either $\mathrm{HH} 24$ experimental occlusions ( $\mathrm{N}=3$ partial occlusions, $\mathrm{N}=1$ full occlusion) or $\mathrm{HH} 24$ in-silico occlusions ( $\mathrm{N}=2$ in-silico occlusion) as time point 2 (Fig. 5). $\mathrm{HH} 18$ to $\mathrm{HH} 24$ change in peak WSS magnitude leads to significant arch artery correlations for each of the arch artery pairs. For control embryos, the majority of WSS-morphology trends were associated with positive slopes, while occlusion embryos were associated with negative slopes, highlighting an overriding of natural PAA growth mechanisms. Pressure pulsatility (the difference between max and min pressure over a cardiac cycle) was also monitored for $\mathrm{HH} 18$ and $\mathrm{HH} 24$ embryos. Significant pulsatility-morphology trends were 
determined for PAA IIIL and IVL. Pulsatility-morphology trends were associated with negative slopes for both control and occlusion embryos.

Local occlusions affect pressure distribution to subsequent circulation. In addition to flow and pressure values within the arches themselves, vessel occlusion also affects flow and pressure distribution curves to subsequent circulation. Upon vessel occlusion, inlet pressure is affected (Fig. 6), despite maintaining the same imposed flow across embryos, with the full occlusion model showing a higher spike than partial occlusion curves for their respective conditions (experimental, in-silico). Pressure curves entering the distal circulations (Windkessel bounds) are largely the same for the left cranial branch, but are slightly elevated for in-silico occlusion and in-silico partial occlusion when compared to its control phenotype (SFig. 4,5).

90-10 cranial caudal flow distributions are largely maintained upon full and partial in-silico vessel occlusion of $\mathrm{HH} 18$ and $\mathrm{HH} 24$ embryos (STable 4) despite bounds not being tuned to maintain the flow split. A slight deviation is seen in the HH24 IVR in-silico occlusion, which has a 89-11 flow split. While changes in the caudal pressure curves are slight, changes in cranial pressures are much larger, particularly for $\mathrm{HH} 18-1$ full occlusion (SFig. 4, magenta). HH24 occluded and partially occluded embryos exhibit a much lower cranial pressure magnitude when compared to that of the control (SFig. 5). Very slight changes are seen in flow curves distributed to the rest of the embryo upon vessel occlusion.

Instantaneous flow changes largely determine vessel functionality. The evolution of pressure and flow values along each arch artery determines its individual 
functionality. Functionality changes are summarized in the form of resistance- area plots (Fig. 6B), which are essentially pressure-drop to flow ratio plotted by average vessel cross-sectional area (CSA). HH24 in-silico occlusion predicted resistance values for three of the remaining five vessels for $\mathrm{HH} 24$ experimental partial occlusions (PAAs VIL, IVL and IIR). Experimental partial occlusion arch arteries underwent an increase or decrease in area to obtain these values. Of the arches not matched by instantaneous (in-silico) flow change predictions, PAA IIIL from the HH24 in-vivo full occlusion model obtained the same area and resistance values as PAA IVR from $\mathrm{HH} 24$ control embryos. PAA IIIL therefore assumed the profile of the normally flow dominant arch in control embryos. PAA VIR from $\mathrm{HH} 24$ in-vivo full occlusion model maintained the same CSA as HH24 in-vivo full occlusion PAA IVL.

A number of vessels maintained the same CSA but different resistances values as peer vessels, emphasizing how CSA does not determine functionality. This is the case for PAA IVR in-silico occlusion and IIIL experimental occlusion. PAA VIL of HH24 in-vivo full occlusion assumed the same cross-sectional area as HH18IVL (control, insilico occlusion/partial occlusion), indicating that IVL did not change morphology between $\mathrm{HH} 18$ and $\mathrm{HH} 24$. HH18 in-silico occlusion/partial occlusion PAA II (R \& L) and PAA IVR share the same CSA value, but resistance values differ largely. These arches are regressing and growing in respectively.

\section{Small initial insult evolves into larger structural deformation. In order to} underscore the importance of the remodeling presented throughout this paper in embryos 24-hours post PAA IVR occlusion, a partial occlusion embryo, Exp-poccl-10, 
was allowed to develop to $\mathrm{HH} 29$ (day 6), when the aortic and pulmonary trunks are clearly distinguishable, the left IV arch artery has regressed, and the aortic trunk has begun to cross under the pulmonary trunk. At HH24 the partial occlusion embryo's stroke volume, cardiac output and ventricular ejection fraction differ slightly from that of HH24 control embryos; only HH24 partial occlusion OFT diameter was statistically significant (two-tailed t-test). By HH26 both the OFT diameter and ventricular ejection fraction of Exp-poccl-10 were statistically different from that of $\mathrm{HH} 26$ controls when using a one-sample T-test. By HH29 morphological differences are readily visible with the poccl geometry displaying hyper-enlarged arches and abnormal rotation of the OFT (Fig. 7, SFig. 6).

\section{Discussion}

We used an experimental-computational approach to quantify morphological and hemodynamic changes along the PAAs following vessel occlusion. Small changes in flow distribution led to significant length, ellipticity, and WSS-morphology trends upon experimental IVR vessel occlusion. In-silico occlusion models captured the instantaneous effects of altered hemodynamics loading, allowing us to propose an adaptive model for PAA morphogenesis detailed below.

Significance

From $\mathrm{HH} 18-\mathrm{HH} 24$, the arch arteries undergo many changes. These changes are the result of inlet flow stream distributions, 3D aortic sac and aortic arch geometries, and local vascular biologic responses to spatial variations in WSS ${ }^{35}$. The early embryo adapted its vasculature using known vascular adaptation mechanisms normally 
associated with the mature vessel. In the mature vascular system, the vascular network works to maintain constant homeostatic stress which consists of shear, axial and circumferential stress along the vessels $6,17,31$. The WSS stimulus leads to diameter changes in response to flow rate ${ }^{26}$. In a 1980 canine carotid artery study, vessel diameter was shown to increase with increased flow load and vice-versa (Kamiya and Togawa $)^{17}$ as a way of bringing shear stress down to control levels. Similarly, a 1987 study observed vessel dilation and no hypertrophy in response to increased flow in the iliac artery of monkeys ${ }^{40}$. HH18 experimental occlusion embryos were also able to adapt their diameters, over the span of 24 hours, to maintain a desired stress state.

Our major findings concern how mechanical interaction between fluid flow in the arches and structural configuration of the vessels influence cardiac outflow morphogenesis. The change in slope of WSS-morphology trends (Fig. 5) underscores a change in growth dynamics upon vessel occlusion. Because flow through these arches are essentially parabolic (Poiseuille flow) and Poiseuille WSS magnitude is proportional to flow over radius cubed $\left(\mathrm{Q} / \mathrm{r}^{3}\right)$ or mean velocity over radius $\left(v_{\text {mean }} / \mathrm{r}\right)$, a positive slope suggests a change in flow/velocity is locally driving WSS change, while a negative slope implies the geometric feature is dominating local WSS change between stages. This indicates that following vessel occlusion, local changes in WSS were associated with remodeling along the arches, contrary to controls where decrease of WSS was rather related to flow. The in-silico occlusion geometries were unsurprisingly dominated by local geometric changes rather than hemodynamics (SFig. 3), as the vessels were constrained to their original configuration. 


\section{Proposed Adaptive Model}

We propose a two-phase adaptation process (Fig. 8) where local increase in pressure accompanied by a decrease in WSS along the occluded vessel ultimately result in observed vessel elongation and CSA changes. Morphological changes identified in Fig 3., indicate that the partial occlusion is causing embryos to resemble that of a more mature phenotype, elongating in size and displaying more rounded crosssectional areas. In the initial phase, immediately following vessel occlusion, a local increase in pressure propagates upstream to the aortic sac accompanied by an increase in WSS. The aortic sac in turn increases in diameter, which causes a decrease in length of the PAAs which are pulled from their aortic sac inlets. The second phase, brought on by the stretching of the arches by the aortic sac, leads to an increase in axial stress, which is compounded by the flow induced asymmetry, culminating in the overall increase in longitudinal length experienced by the arches. Concurrent with the phase 1's local increase in pressure, is a decrease in WSS experienced by the occlusion branch which leads to a shrinking or altogether elimination of the arch and adds to the flow induced asymmetry. In this way, natural growth mechanisms are overridden and the would be presumptive aortic arch is prevented from becoming the dominant arch of the aorta.

\section{Critical Window of Development}

The $\mathrm{HH} 18$ time point is a particularly critical stage of cardiovascular development as it precedes migration of neural crest cells down the pharyngeal arches. Following formation of arch arteries III, IV, VI, neural crest cells seed the arch arteries, covering their endothelial sheath and making up their ectomesenchyme ${ }^{19}$. A subset of these 
cells reach the outflow tract cushions by HH23. Cardiac neural crest cells (CNCCs), along with a shelf of tissue from the aortic sac, form the aortopulmonary septation complex and initiate septation ${ }^{34}$. Disruption of this process due to altered mechanical loads within the vessels would lead to a variety of cardiac abnormalities. CNCC ablations are associated with failure of arch arteries three, four (right), and six to develop to the proper size ${ }^{2}$. There is a loss of bilateral symmetry, with uncommonly small or collapsed arch arteries on one side and unusually large arteries on the opposite side ${ }^{33}$. Many of these phenotypes can be seen in the experimental occlusion geometries found in Supplemental Figure 7.

Typically, asymmetrical remodeling begins at $\mathrm{HH} 28 / \mathrm{E} 12.5^{10,11}$, with the left fourth arch artery regressing in the chick embryo and the right fourth forming the proximal part of the subclavian artery in the mouse embryo. The experimental late stage (HH29) partial occlusion embryo, Exp-poccl-10, did not undergo this asymmetric remodeling. Its phenotype approaches that of the Pitx $2 \delta c$ mutant hearts with transposition of the great arteries, where the aortic and pulmonary trunk remain parallel. Pitx2 regulates left-right asymmetry to asymmetrically developing organs and is associated with patterning of the OFT myocardium $22,23,32$. This myocardium is derived from the pharyngeal mesoderm. Between Stages $\mathrm{HH} 21$ and $\mathrm{HH} 30$, Carnegie stages 15 and 19, the OFT-PAA junction undergoes rotation in the counterclockwise direction ${ }^{1,24}$. Observed rotation may be the result of asymmetrical lengthening of the right ventricular OFT, which causes the pulmonary trunk to be "pushed" towards its definitive position ${ }^{28}$. Here we show that this defect may be the result of abnormal flow patterning originating from the PAAs themselves. 


\section{Limitations:}

A small subset of embryos underwent computational modeling. While this is typical in computational studies, biological studies generally have larger sample sizes. We believe the study's purpose of characterizing the natural variation in altered mechanical loading still holds and the necessary representations have been presented. Detailed 3D reconstructions of the experimental occlusions allowed for a more thorough probe into the embryos' adaptive response than that of our previous study ${ }^{21}$. Results led us to propose a two-phase adaptation response. This two-phase adaptation hypothesis needs to be confirmed by further experiments that could include continuous imaging of the PAA manifold or labeling of different arch sections so as to track respective lengthening. Outlet boundary conditions and flow input curves were taken to be the same for both control and occlusion models. Computational simulations were validated for control embryos ${ }^{21}$, and assumed to remain valid for occlusion embryos. It is possible the occluded embryos may be physiologically disturbed such that their input flow profiles are altered from that of controls.

Conclusions:

This study demonstrates that abnormal PAA hemodynamics can precipitate abnormal cardiac function given the correct timing and location of injury. In their 2007 study, Yashiro et al. showed that differential flow patterns and abnormal PAA remodeling can result from abnormal spiraling of the OFT. Following Pitx2 induction of abnormal OFT morphology, differential flow patterns led to abnormal molecular expression patterns and abnormal PAA VI patterning in E11 -11.5 mice $(\mathrm{HH} 18-\mathrm{HH} 24$ equivalent $)^{38}$. Here, we show the connection between abnormal PAA IV patterning and 
OFT morphology, with the defect originating from within the PAAs themselves.

Following arch artery occlusion, local changes in WSS are associated with geometric remodeling along the entire PAA manifold, while pressure changes are largely confined to the aortic sac. Wall shear stress drives defect propagation which can result in enlarged OFT diameters, irregular regression patterns and abnormal rotation of the OFT. Position and length of the initial occlusion insult impact subsequent remodeling defects. While both full and partial occlusions led to an overriding of natural growth mechanisms, partial occlusions were able to maintain a lower per arch average pressure and WSS distribution than that of its full occlusion counterpart. Importantly, partial occlusion embryos were able to fully dissipate WSS and pressure fluctuations laterally along the length of the arches before the dorsal aorta in contrast to the full vessel occlusion embryo. This work is the first step in understanding the effect of varying degrees of PAA occlusion in what is programmed to be the dominant arch of the aorta. By examining embryo-specific occlusion geometries in 3D and comparing them to that of in-silico occlusion embryos, we distinguish immediate changes from that of longer-term adaptation to changing hemodynamic conditions and show how the former influences the later. In identifying spatial-temporal markers of abnormal development, this work can be used to identify new genes and biochemical compounds that affect cardiac outflow morphogenesis.

\section{Acknowledgements:}

We thank the Cornell BRC imaging facility and Randolph Linderman (reconstructions). This work was funded by NSF GRFP, NSF GROW, an Alfred P. Sloan Foundation 
fellowship, CMMI-1635712, an Inria International Internship grant and by the National Institutes of Health (HL110328, S10OD012287 and S10OD016191).

\section{Disclosures:}

The authors have no disclosures.

\section{References:}

1. Bajolle, F., S. Zaffran, R. G. Kelly, J. Hadchouel, D. Bonnet, N. A. Brown, and M. E. Buckingham. Rotation of the myocardial wall of the outflow tract is implicated in the normal positioning of the great arteries. Circ. Res. 98:421-428, 2006.

2. Bockman, D. E., M. E. Redmond, K. Waldo, H. Davis, and M. L. Kirby. Effect of neural crest ablation on development of the heart and arch arteries in the chick. Am. J. Anat. 180:332-41, 1987.

3. De Clercq, K., E. Persoons, T. Napso, C. Luyten, T. N. Parac-Vogt, A. N. Sferruzzi-Perri, G. Kerckhofs, and J. Vriens. High-resolution contrast-enhanced microCT reveals the true three-dimensional morphology of the murine placenta. Proc. Natl. Acad. Sci. U. S. A. 116:13927-13936, 2019.

4. deAlmeida, A., T. McQuinn, and D. Sedmera. Increased ventricular preload is compensated by myocyte proliferation in normal and hypoplastic fetal chick left ventricle. Circ. Res. 100:1363-70, 2007.

5. Fisher, A. B., S. Chien, A. I. Barakat, and R. M. Nerem. Endothelial cellular response to altered shear stress. Am. J. Physiol. Lung Cell. Mol. Physiol. 281:L529-33, 2001.

6. Girerd, X., G. London, P. Boutouyrie, J.-J. Mourad, M. Safar, and S. Laurent. Remodeling of the Radial Artery in Response to a Chronic Increase in Shear 
Stress. Hypertension 27:799-803, 1996.

7. Go, A. S. et al. Heart disease and stroke statistics--2013 update: a report from the American Heart Association. Circulation 127:e6-e245, 2013.

8. Harh, J. Y., M. H. Paul, W. J. Gallen, D. Z. Friedberg, and S. Kaplan. Experimental production of hypoplastic left heart syndrome in the chick embryo. Am. J. Cardiol. 31:51-6, 1973.

9. Van der Heiden, K., B. C. W. Groenendijk, B. P. Hierck, B. Hogers, H. K. Koerten, A. M. Mommaas, A. C. Gittenberger-de Groot, and R. E. Poelmann. Monocilia on chicken embryonic endocardium in low shear stress areas. Dev. Dyn. 235:19-28, 2006.

10. Hiruma, T., and R. Hirakow. Formation of the pharyngeal arch arteries in the chick embryo. Observations of corrosion casts by scanning electron microscopy. Anat. Embryol. (Berl). 191:415-23, 1995.

11. Hiruma, T., Y. Nakajima, and H. Nakamura. Development of pharyngeal arch arteries in early mouse embryo. J. Anat. 201:15-29, 2002.

12. Hogers, B., M. C. C. DeRuiter, A. C. C. Gittenberger-de Groot, and R. E. E. Poelmann. Unilateral vitelline vein ligation alters intracardiac blood flow patterns and morphogenesis in the chick embryo. Circ. Res. 80:473-481, 1997.

13. Hogers, B., M. C. DeRuiter, a C. Gittenberger-de Groot, and R. E. Poelmann. Extraembryonic venous obstructions lead to cardiovascular malformations and can be embryolethal. Cardiovasc. Res. 41:87-99, 1999.

14. Hove, J. R., R. W. Köster, A. S. Forouhar, G. Acevedo-Bolton, S. E. Fraser, and M. Gharib. Intracardiac fluid forces are an essential epigenetic factor for 
embryonic cardiogenesis. Nature 421:172-7, 2003.

15. Hu, N., D. a Christensen, A. K. Agrawal, C. Beaumont, E. B. Clark, and J. a Hawkins. Dependence of aortic arch morphogenesis on intracardiac blood flow in the left atrial ligated chick embryo. Anat. Rec. (Hoboken). 292:652-60, 2009.

16. Hu, N., and E. Clark. Hemodynamics of the stage 12 to stage 29 chick embryo. Circ. Res. 65:1665-1670, 1989.

17. Kamiya, A., and T. Togawa. Adaptive regulation of wall shear stress to flow change in the canine carotid artery. Am. J. Physiol. - Hear. Circ. Physiol. 8:14-21, 1980.

18. Kowalski, W. J., O. Dur, Y. Wang, M. J. Patrick, J. P. Tinney, B. B. Keller, and K. Pekkan. Critical Transitions in Early Embryonic Aortic Arch Patterning and Hemodynamics. PLoS One 8:e60271, 2013.

19. Kuratani, S. C., and M. L. Kirby. Initial migration and distribution of the cardiac neural crest in the avian embryo: an introduction to the concept of the circumpharyngeal crest. Am. J. Anat. 191:215-27, 1991.

20. Lindsey, S. E., J. T. Butcher, and I. E. Vignon-Clementel. Cohort-based multiscale analysis of hemodynamic-driven growth and remodeling of the embryonic pharyngeal arch arteries. Development 145:, 2018.

21. Lindsey, S. E., P. G. Menon, W. J. Kowalski, A. Shekhar, H. C. Yalcin, N. Nishimura, C. B. Schaffer, J. T. Butcher, and K. Pekkan. Growth and hemodynamics after early embryonic aortic arch occlusion. Biomech. Model. Mechanobiol. 14:735-751, 2015.

22. Liu, C., W. Liu, M. F. Lu, N. A. Brown, and J. F. Martin. Regulation of left-right 
asymmetry by thresholds of Pitx2c activity. Development 128:2039-2048, 2001.

23. Liu, C., W. Liu, J. Palie, M. F. Lu, N. A. Brown, and J. F. Martin. Pitx2c patterns anterior myocardium and aortic arch vessels and is required for local cell movement into atrioventricular cushions. Development 129:5081-5091, 2002.

24. Lomonico, M. P., G. W. Moore, and G. M. Hutchins. Rotation of the junction of the outflow tract and great arteries in the embryonic human heart. Anat. Rec. 216:544-549, 1986.

25. Pant, S., B. Fabrèges, J. F. Gerbeau, and I. E. Vignon-Clementel. A multiscale filtering-based parameter estimation method for patient-specific coarctation simulations in rest and exercise. Lect. Notes Comput. Sci. 8330 LNCS:102-109, 2014.

26. Pries, A. R., T. W. Secomb, and P. Gaehtgens. Structural adaptation and stability of microvascular networks: Theory and simulations. Am. J. Physiol. Hear. Circ. Physiol. 275:349-360, 1998.

27. Rychter, Z. Experimental morphology of the aortic arches and the heart loop in chick embryos. Adv Morphog , 1962.

28. Scherptong, R. W. C., M. R. M. Jongbloed, L. Wisse, J., R. Vicente-Steijn, M. M. Bartelings, R. E. Poelmann, M. J. Schalij, and A. C. Gittenberger-de Groot. Morphogenesis of Outflow Tract Rotation During Cardiac Development: The Pulmonary Push Concept. Dev. Dyn. 241:1413-1422, 2012.

29. Sedmera, D., T. Pexieder, V. Rychterova, N. Hu, and E. B. Clark. Remodeling of chick embryonic ventricular myoarchitecture under experimentally changed loading conditions. Anat. Rec. 254:238-52, 1999. 
30. Tobita, K., and B. B. Keller. Right and left ventricular wall deformation patterns in normal and left heart hypoplasia chick embryos. Am. J. Physiol. Heart Circ. Physiol. 279:H959-69, 2000.

31. Valentin, A., L. Cardamone, S. Baek, and J. D. Humphrey. Mechanical stress exerts control over cellular behavior in the embryo. Biomechanical forces connect genetic and molecular-level events to tissue-level deformations. J. R. Soc. Interface 6:293-306, 2009.

32. Waldo, K. L., M. R. Hutson, C. C. Ward, M. Zdanowicz, H. A. Stadt, D. Kumiski, R. Abu-Issa, and M. L. Kirby. Secondary heart field contributes myocardium and smooth muscle to the arterial pole of the developing heart. Dev. Biol. 281:78-90, 2005.

33. Waldo, K. L., D. Kumiski, and M. L. Kirby. Cardiac neural crest is essential for the persistence rather than the formation of an arch artery. Dev. Dyn. 205:281-92, 1996.

34. Waldo, K., S. Miyagawa-Tomita, D. Kumiski, and M. L. Kirby. Cardiac neural crest cells provide new insight into septation of the cardiac outflow tract: aortic sac to ventricular septal closure. Dev. Biol. 196:129-44, 1998.

35. Wang, Y., O. Dur, M. J. Patrick, J. P. Tinney, K. Tobita, B. B. Keller, and K. Pekkan. Aortic arch morphogenesis and flow modeling in the chick embryo. Ann. Biomed. Eng. 37:1069-81, 2009.

36. Wyczalkowski, M. A., Z. Chen, B. A. Filas, V. D. Varner, and L. A. Taber. Computational models for mechanics of morphogenesis. Birth Defects Res. C. Embryo Today 96:132-52, 2012. 
37. Yalcin, H. C., A. Shekhar, T. C. McQuinn, and J. T. Butcher. Hemodynamic patterning of the avian atrioventricular valve. Dev. Dyn. 240:23-35, 2011.

38. Yashiro, K., H. Shiratori, and H. Hamada. Haemodynamics determined by a genetic programme govern asymmetric development of the aortic arch. Nature 450:285-8, 2007.

39. Yoshigi, M., G. D. Knott, and B. B. Keller. Lumped parameter estimation for the embryonic chick vascular system: a time-domain approach using MLAB. Comput. Methods Programs Biomed. 63:29-41, 2000.

40. Zarins, C. K., M. A. Zatina, D. P. Giddens, D. N. Ku, and S. Glagov. Shear stress regulation of artery lumen diameter in experimental atherogenesis. J. Vasc. Surg. 5:413-420, 1987. 
Figure 1: Reference Guide for Cardiac Development and Experimental Pipeline. A) Timeline of critical cardiac structural events with arch numbers and configurations listed for stages examined in this study (white arrow). B) Highly targeted occlusion experiment led to changes in hemodynamics. In-silico occlusion experiments allowed for high resolution pressure and WSS maps. Combining immediate flow changes with known adaptation phenomenon allowed for the prediction of morphological changes. Theoretical predictions were compared with experimental geometries at later stages. HH18 pressure maps are shown for in-silico occlusions (occlusions indicated by gray arrows) while WSS maps are shown for $\mathrm{HH} 24$ experimental occlusions.

Figure 2. HH24 hemodynamic changes resulting from experimental vessel occlusion. (A) Flow distributions per arch (+/-s.e.) asterisks indicate a statistically significant change for occlusions embryos when compared to controls using a two tailed student $t$ (poccl, in-silico $\mathrm{occl} / \mathrm{poccl}$ ) or one-sample t-test (occl) (B) Pressure and wall shear stress $\left(\right.$ dynes $\left./ \mathrm{cm}^{2}\right)$ at peak flow for a representative $\mathrm{HH} 24$ control embryo (top) and each of the partial occlusion geometries in which CFD was run. Although the geometries themselves have changed, partial occlusion pressure and WSS maps resemble that of $\mathrm{HH} 24$ control embryos (see ${ }^{20}$ for range of variability). Only the full occlusion geometry's pressure is so high that it cannot be fully dissipated along the arches and a high-pressure zone is carried over to the DoA. $(\mathrm{N}=5 \mathrm{cntrl}, \mathrm{N}=3 \exp$ poccl, $\mathrm{N}=1 \exp$ occl, $\mathrm{N}=2$ in-silico occl; in-silico poccl) 


\section{Figure 3. HH24 Occlusion Embryo Morphological Changes Measured from 3D}

Reconstructions. Ellipticity changes for both the HH24 control and HH24 partial occlusion (poccl) geometries (A) Control and partial occlusion templates showing the average trend along with vessel cross-sections where control is displayed in black and overlayed over poccl crosssections (B) Boxplots detail changes for each of the ten sections along the centerline of each arch (cntrl, poccl), single marker used for full occlusion $(\mathrm{N}=1)$. (C) PAA length per arch with asterisks indicating a statically significant difference when considering a two-tailed paired t-test. $(\mathrm{N}=3$, poccl, $\mathrm{N}=5 \mathrm{cntrl})$. Occlusion geometries have more circular arch cross-sections and more elongated arches. D) Cross-sectional area (CSA) for the control and poccl templates shown (top) with box-plots that detail the changes taking place along the centerline (' $\mathrm{T}$ ' represents extreme bounds,'+' outliers). Single marker used for full occlusion. The largest CSA changes are apparent in PAA III, particularly PAA IIIL and PAA IV. (E) branch-splitting angles with mean values for $\mathrm{HH} 24$ control displayed as dashed lines along corresponding arches for both $\mathrm{HH} 24$ partial occlusion (po) and full occlusion (o). The largest differences can be seen for PAA III, though no significant changes were found $(\mathrm{N}=5 \mathrm{cntrl}, \mathrm{N}=3$ poccl, $\mathrm{N}=1$ occl).

Figure 4. HH18 HH24 in-silico occlusion hemodynamic changes (A) OD resistance values for each arch per stage. The only significant difference found when comparing HH18 controls to insilico occlusion or HH24 experimental occlusions to HH24 occlusions was for HH18 PAA IVR (HH18 to HH18So, two-tailed two-sample t-test). (B) Pressure and WSS (dynes $/ \mathrm{cm}^{2}$ ) maps at peak inflow for a re-presentative HH18 and HH24 in-silico occlusion geometry. Note how HH18 in-silico occlusion effects are subtle due to the small amount of flow being re-distributed, while HH24 effects are more pronounced for both full a partial occlusion, through the difference 
between degree of occlusion is hard to distinguish. So, Spo = in-silico occlusion, partial occlusion, respectively. ( $\mathrm{N}=5$ cntrl, $\mathrm{N}=3$ exp poccl, $\mathrm{N}=1$ exp occl, $\mathrm{N}=2$ in-silico occl; insilico poccl).

Figure 5. Occlusion driven geometric changes. Linear regressions plotted to highlight correlations between hemodynamic forces (peak WSS and pressure pulsatility) and area diameter changes that occur with occlusion insult. Axes show changes in $\mathrm{x}$ and changes in y values. Only significant trends are shown. (circles indicate right side, squares, left side). Control curves shown in blue, when significant. $(\mathrm{N}=5 \mathrm{cntrl}, \mathrm{N}=3$ poccl, $\mathrm{N}=1 \mathrm{occl})$.

Figure 6. Pressure and functionality changes upon vessel occlusion (A) 3D-0D resulting inlet pressure curves (top) and DoA branch (R \& L) outlet curves for each of the occlusion conditions, both experimental and in-silico. Standard error bars are shown for each curve. (B) CFD-based resistance-area graph highlights vessel functionality. R-A axes show current $\mathrm{x}$ values with current y values. Standard error bars are shown in black. Note how HH24 in-silico occlusion predicted resistance values for three of the remaining five vessels for $\mathrm{HH} 24$ experimental partial occlusions. HH24 IIIL of the in-vivo full occlusion took on the same resistance and area values as HH24 IVR controls. PAA VIL of HH24 in-vivo full occlusion took on the same cross area as HH18IVL. ( $\mathrm{N}=5$ cntrl, $\mathrm{N}=3$ exp poccl, $\mathrm{N}=1$ exp occl, $\mathrm{N}=2$ in-silico occl; insilico poccl).

Figure 7. Later stage partial occlusion phenotype (A) Top down view of PAA manifold of HH26 -HH31 embryos with controls in grey and partial occlusion in peach. Asterisks indicate 
that the embryo is left-sided. Geometries are not shown to scale. Of note is the presence of six arch arteries in the HH29 partial occlusion manifold, when five are present in control counterparts. The enlarged arch arteries of the aortic and pulmonary trunks run parallel to each other along the dorsal-ventral axis in contrast to their control counterparts whose aorta and pulmonary trunks are offset allowing for the aortic trunk to pass under the pulmonary trunk. B) $\mathrm{HH} 24$ diameter cardiac output, stroke volume, ventricular ejection fraction $(\mathrm{n}=5$ control, $\mathrm{n}=3$ poccl, $\mathrm{n}=1$ occlusion). Statistically significant differences were found for OFT diameter changes and when comparing the occlusion phenotype to controls using a one sample t-test. The highlighted poocl from (A) is among poocl values. (C) HH26 diameter and ejection fraction for HH26 embryos ( $\mathrm{n}=5$ control, $\mathrm{n}=1$ poccl). Statistically significant differences were only found when comparing the occlusion phenotype to controls using a one sample t-test. The highlighted poocl from (A) is among poocl values.

Figure 8. Two-phase vessel adaptation mechanism following vessel occlusion. Local and upstream pressure changes lead to WSS changes that ultimately shape the structural changes seen in experimental occlusion embryos. Changes in aortic sac diameter lead to increased axial stress and accounts for the longitudinal lengthening of the arch arteries. 
Experimental 24 hours post-

Occlusion occlusion

(PAA IVR) period
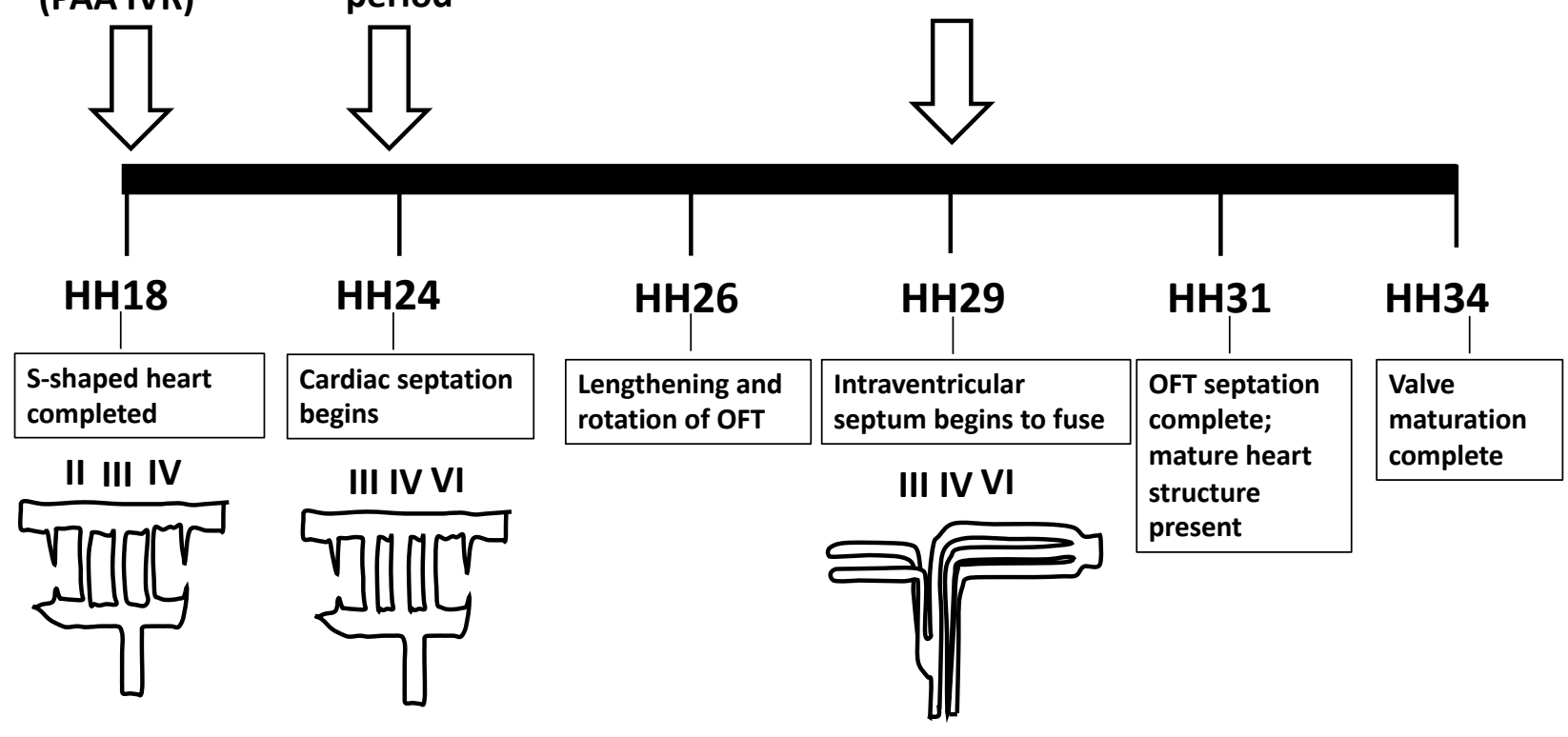

\section{B) Experimental-Computational Pipeline}

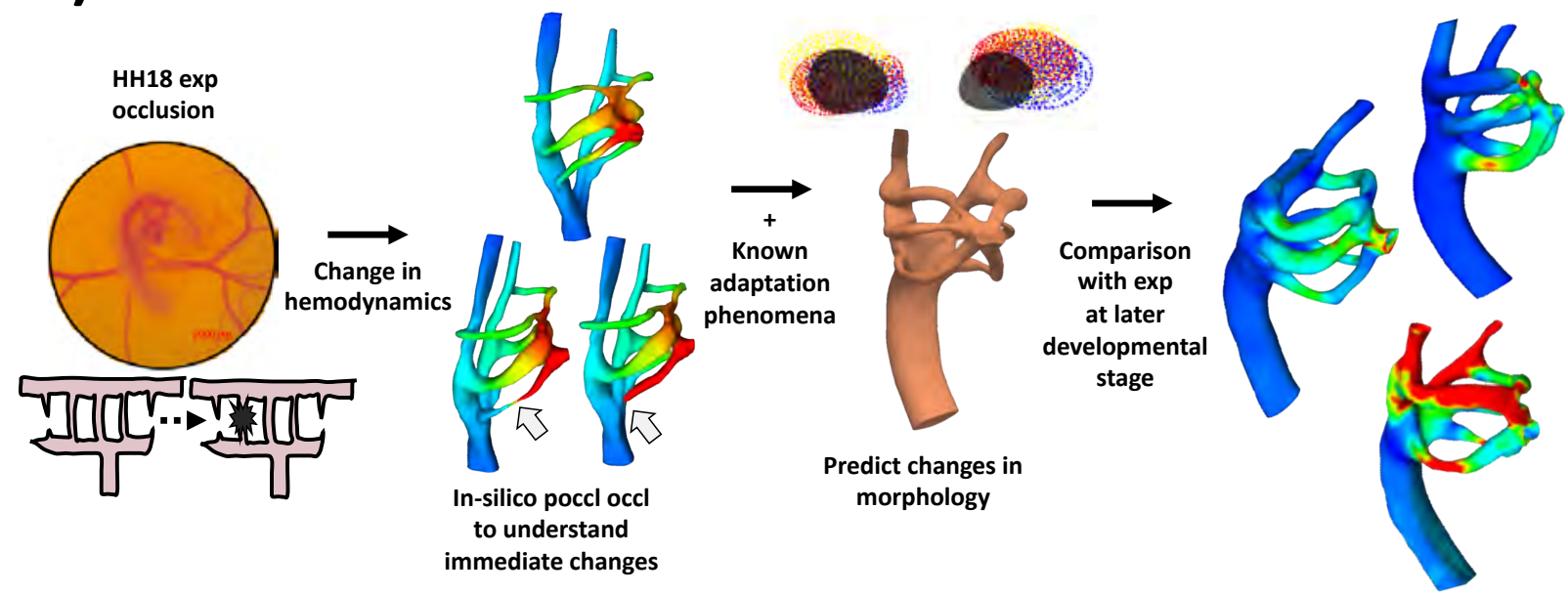


A)

Flow Distribution Arch Arteries [\%]
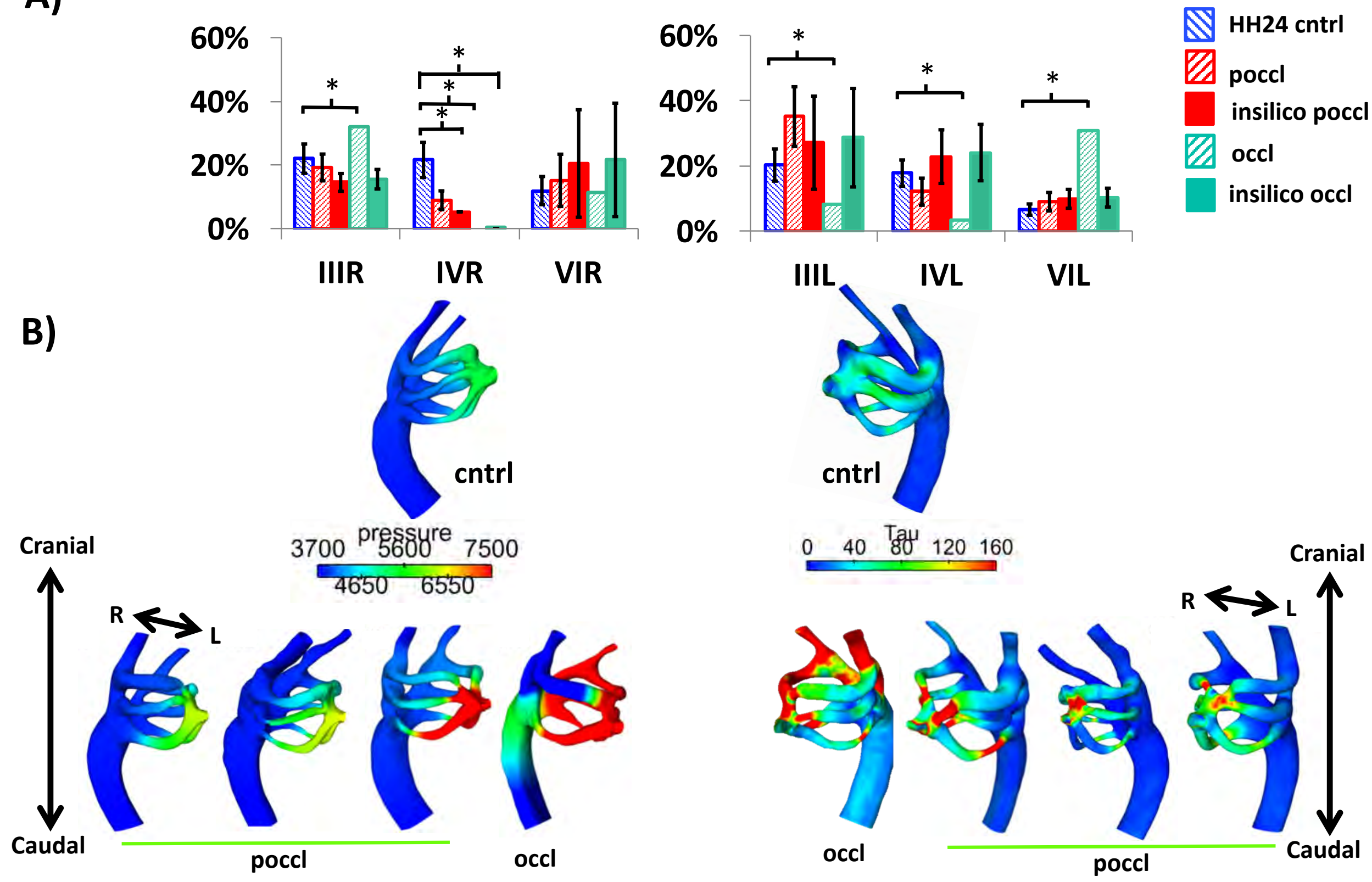
A)

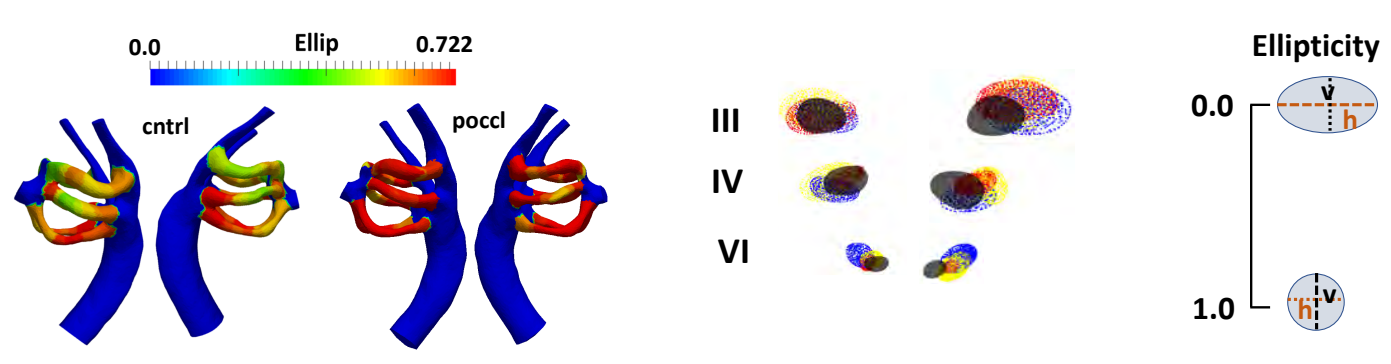

B)

- occl $\square$ cntrl $\square$ poccl C) Curved Arch Artery Centerline Length [cm]

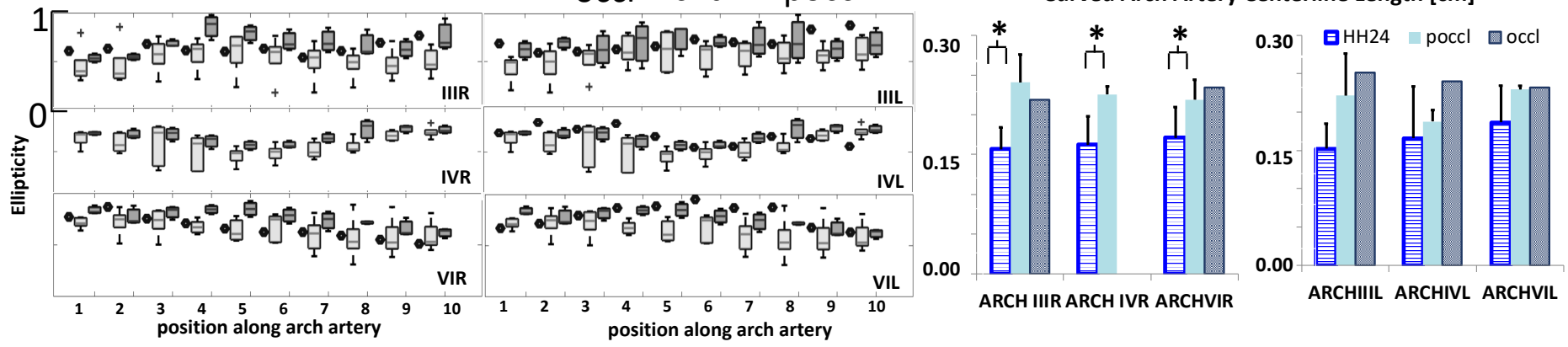

D)

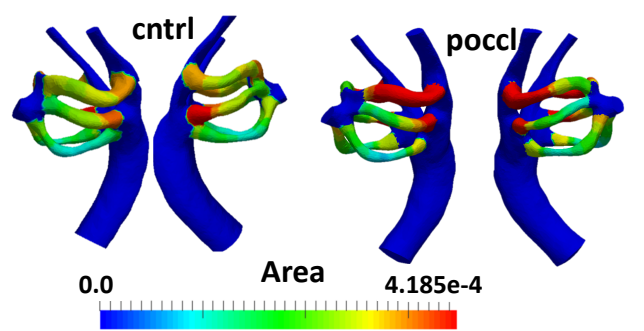

E)
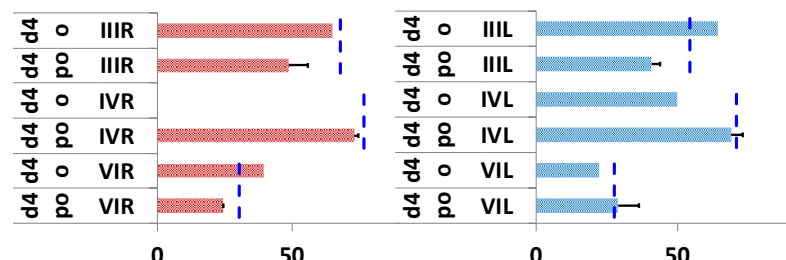

- occl $\square$ cntrl $\square$ pocc

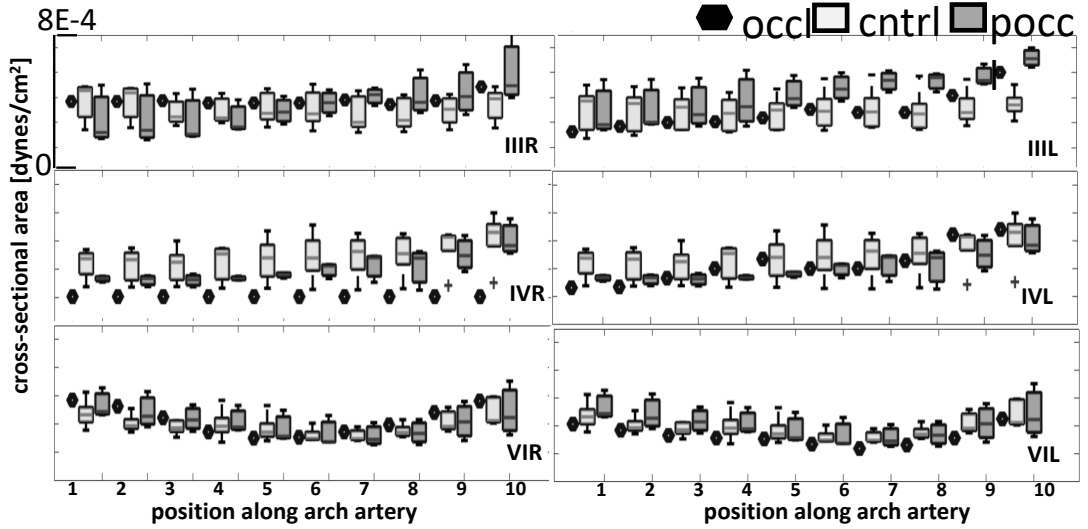

$45 \square \mathrm{HH} 24$ poccl 圆 H 24 occl

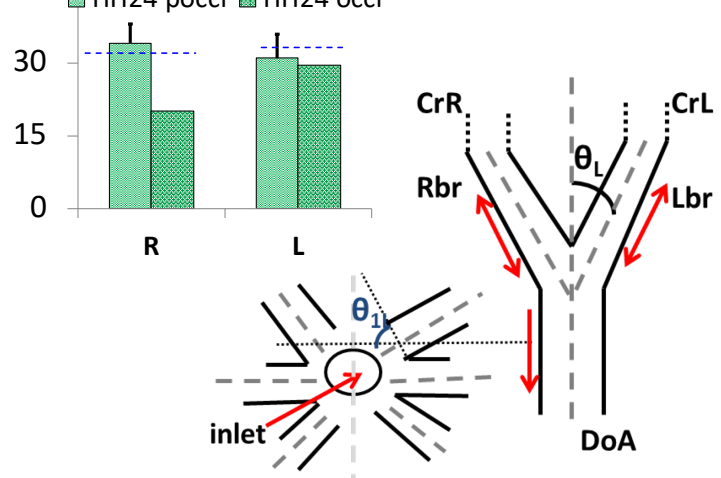




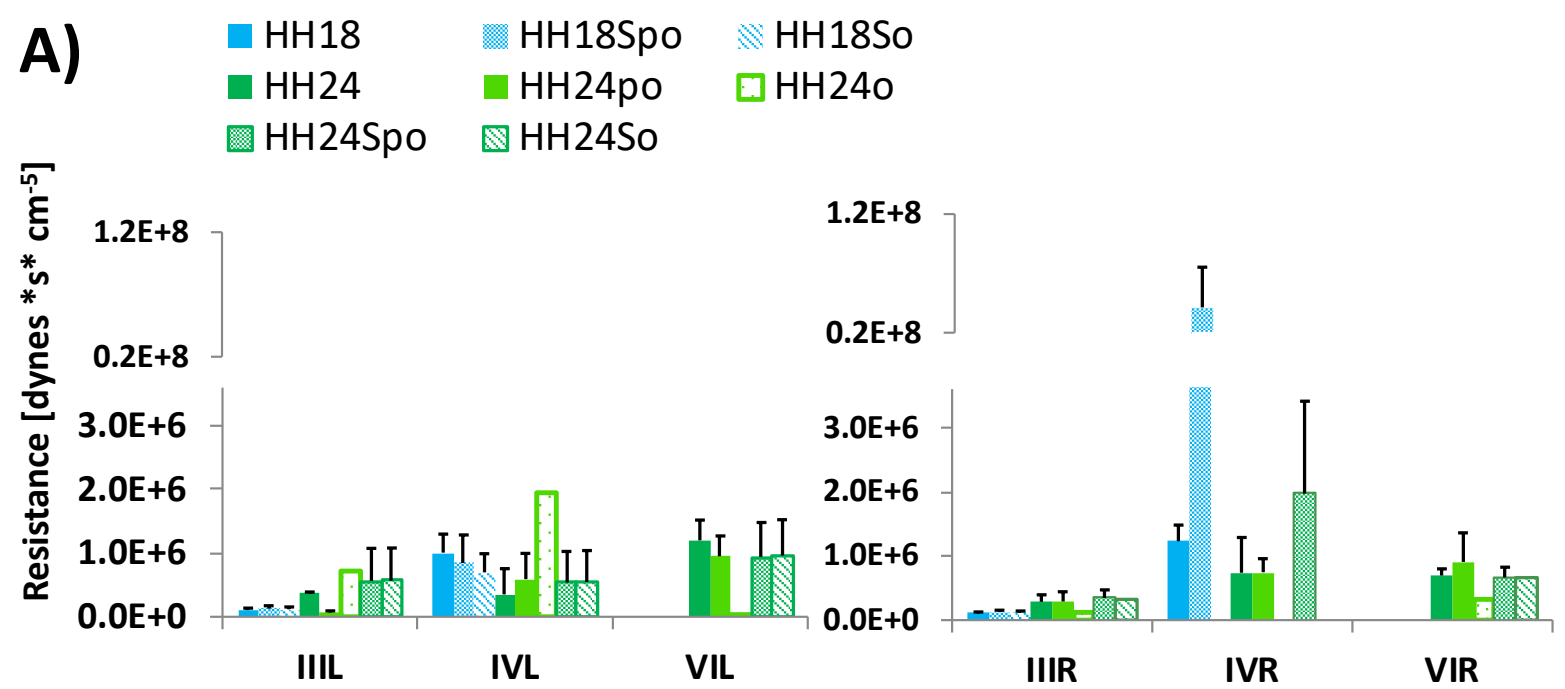

B)
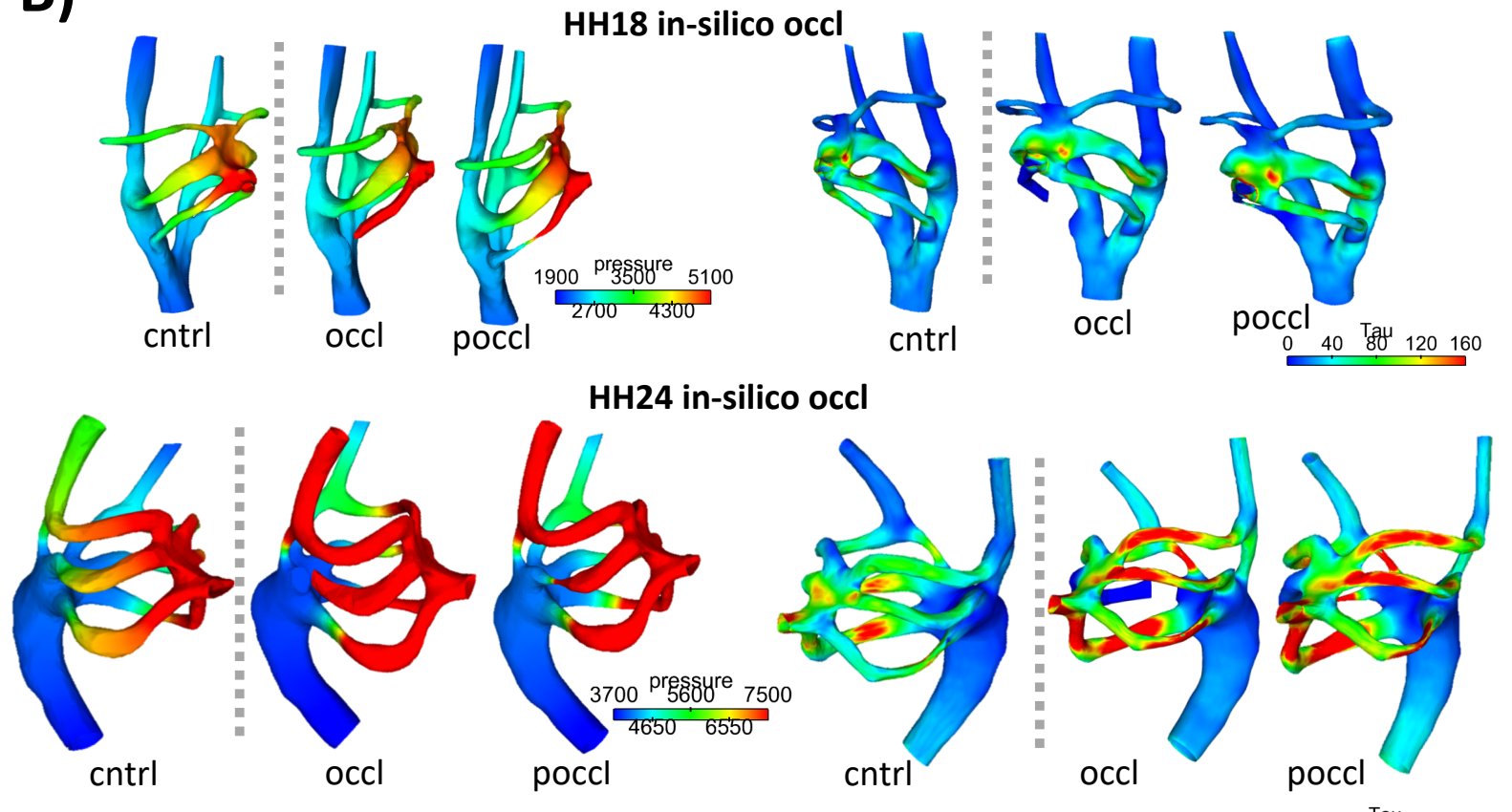

\begin{tabular}{l}
40 T. 80120160 \\
\hline
\end{tabular} 
Significant WSS-morphology trends
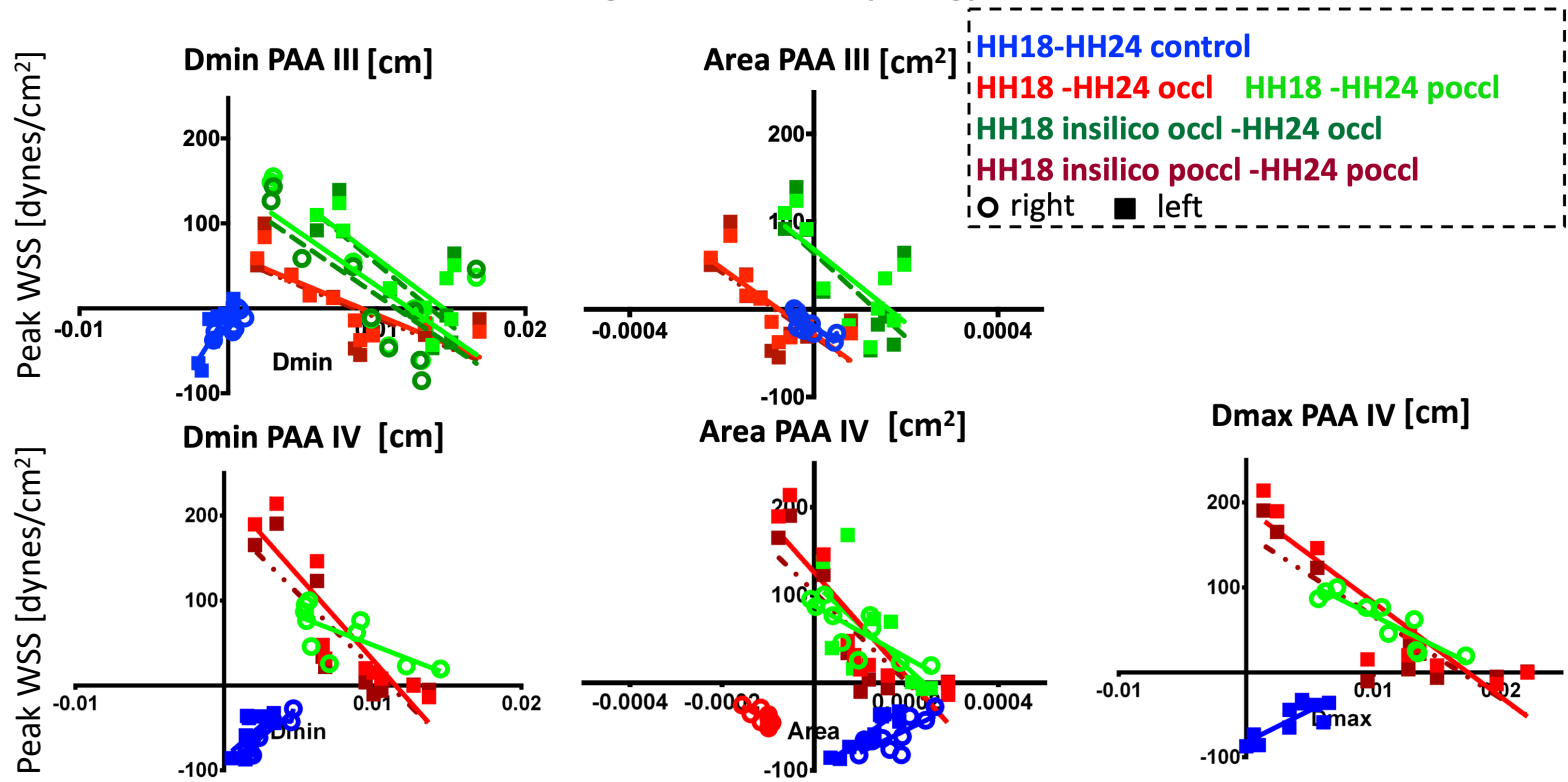

Significant pulsatility-morphology trends

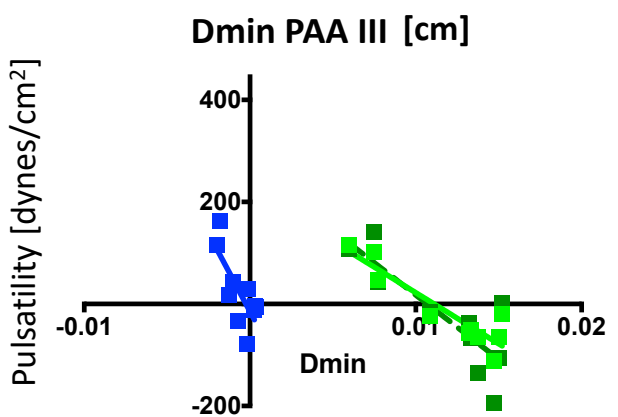

Area PAA III $\left[\mathrm{cm}^{2}\right]$
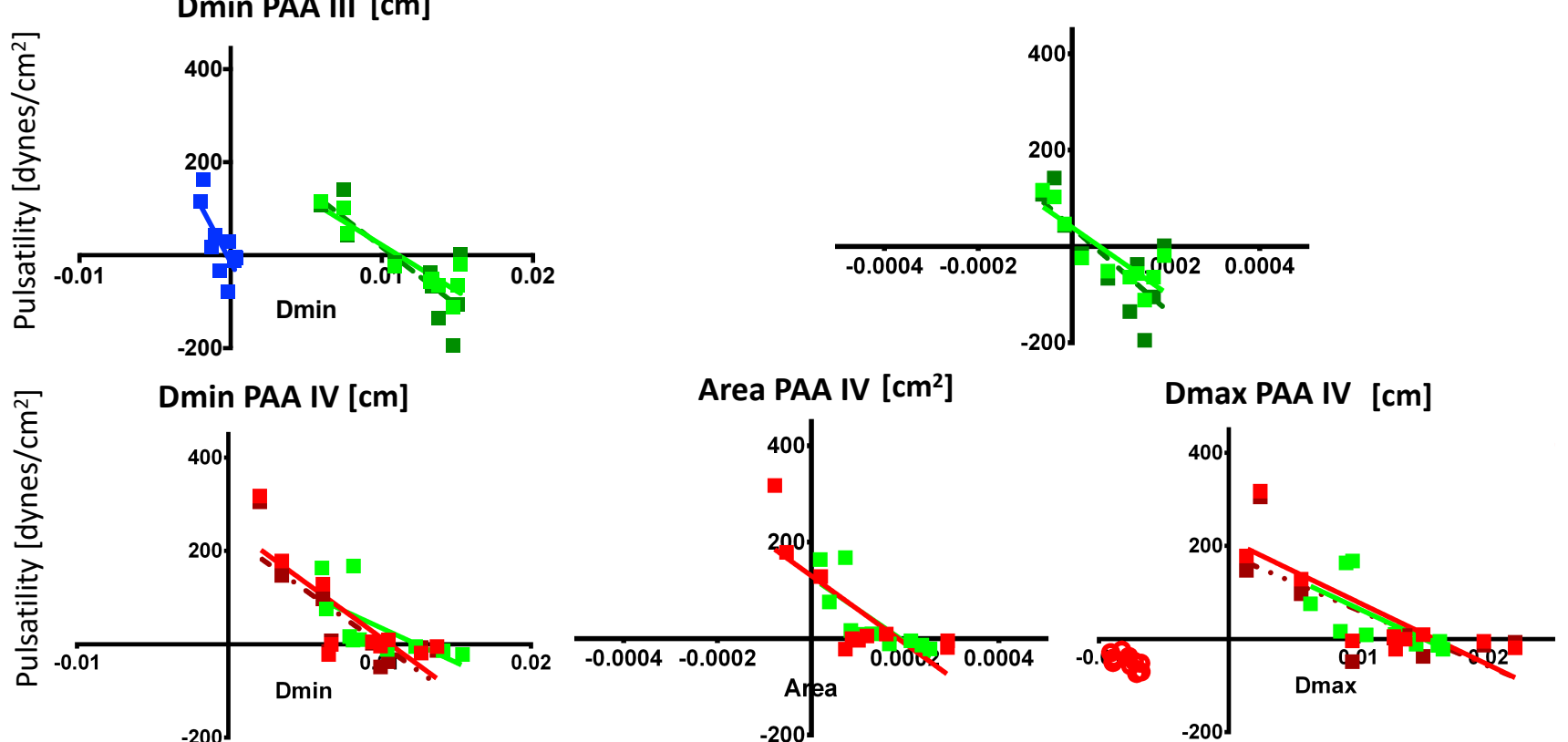
